\title{
Mass hierarchies and dynamical field range
}

\author{
Aitor Landete ${ }^{*}$ and Gary Shiu ${ }^{\dagger}$ \\ Department of Physics, University of Wisconsin-Madison, Madison, Wisconsin 53706, USA
}

(Received 24 July 2018; published 21 September 2018)

\begin{abstract}
Several swampland conjectures suggest that there is a critical field range beyond which the effective field theory (EFT) description breaks down in quantum gravity. In applications of these conjectures, however, the field range of interest is the field space distance traced by the physical trajectory that solves the equations of motion. We refer to this field space distance as the dynamical field range. We show that in the absence of a mass hierarchy between the light and heavy fields, the trajectory of the light field does not, in general, follow a geodesic in field space. Then, stabilizing the heavy fields at the minimum of their potential does not accurately describe the dynamics of the light field in general. A mass hierarchy can delay the breakdown of the EFT and extend the effective field range. We illustrate these subtleties of multifield dynamics with axions in type II string compactifications.
\end{abstract}

DOI: 10.1103/PhysRevD.98.066012

\section{INTRODUCTION}

In recent years, an increasing amount of evidence suggests that not every low-energy effective field theory (EFT) can be consistently coupled to quantum gravity. The quantum gravity constraints that have been unveiled so far have many ramifications. For instance, studies of the generic properties of quantum gravity point to a restriction on the allowed field range in ultraviolet (UV) complete theories. This restriction takes various forms. The weak gravity conjecture [1] asserts that for every long range force, there exists a state whose charge-to-mass ratio is bigger than that of an extremal black hole. This implies upon dualizing an axionlike field to a $U(1)$ gauge field that the axionic decay constant, $f$, is limited by $f \cdot S_{\text {inst }}<$ $\mathcal{O}(1) M_{P}$ [2,3], where $S_{\text {inst }}$ is the instanton action that controls the nonperturbative breaking of the shift symmetry. A more general statement about field ranges, known as the swampland distance conjecture (SDC), was put forth in [4]. This conjecture is based on the observation that in known string constructions, a tower of states become exponentially light as we traverse a large distance $d\left(p_{0}, p\right)$ in field space:

$$
M \sim M_{0} e^{-\lambda d\left(p, p_{o}\right)},
$$

where $\lambda$ is some unspecified positive constant, and thus the EFT breaks down beyond a critical distance $d\left(p_{0}, p\right)>\lambda^{-1}$.

\footnotetext{
*aitor.landete@wisc.edu

†shiu@physics.wisc.edu
}

Published by the American Physical Society under the terms of the Creative Commons Attribution 4.0 International license. Further distribution of this work must maintain attribution to the author(s) and the published article's title, journal citation, and DOI. Funded by SCOAP ${ }^{3}$.
For recent considerations about this conjecture, we refer the reader to $[5,6]$.

The conjectured restrictions on the allowed field range, if proven, have wide-ranging implications, one of which concerns the amplitude of gravitational waves generated by inflation. A kinematic bound due to Lyth [7] relates observable tensor modes to super-Planckian field displacements:

$$
\frac{\Delta \phi}{M_{\mathrm{Pl}}} \gtrsim \mathcal{O}(1) \times \sqrt{\frac{r}{0.01}},
$$

where $r$ is the tensor-to-scalar ratio. Thus, if quantum gravity can impose a strict upper bound on the inflaton field range, one may gain a better certainty on our target for $r$. Besides the challenge in maintaining control of the EFT over a superPlanckian field displacement, a detectable level of tensor modes also poses an additional challenge on the UV completion of inflation. This is because for large-field inflation (say, $r \gtrsim 10^{-2}$ ), the corresponding Hubble scale is $H \sim 10^{14} \mathrm{GeV}$ [8]. As UV complete theories of gravity typically involve new degrees of freedom below the scale of quantum gravity, it is a formidable task to pack the associated energy scales in between $H$ and $M_{\mathrm{Pl}}$. To be concrete, constructing single-field inflation models from string theory requires a hierarchy of scales:

$$
M_{\varphi}<H<M_{\text {moduli }}<M_{\mathrm{KK}}<M_{s}<M_{\mathrm{Pl}},
$$

where $M_{\varphi}$ is the inflaton mass, $M_{\text {moduli }}$ denotes generically the mass scale of the stabilized moduli, $M_{K K}$ refers to the mass of the lightest Kaluza-Klein replica, and $M_{s}$ is the string scale. The challenge in maintaining this hierarchy is thus twofold: (1) the moduli stabilization mechanism should generate this separation of scales (see, e.g., [9] for a 
discussion of this issue in flux compactifications), and (2) backreaction of the heavy fields (which in general has the effect of flattening the inflaton potential $[10,11]$ ) throughout the inflationary trajectory should be consistently taken into account.

Given the above considerations, it is important to define a proper measure of the maximum field range. The main goal of this paper is to make clear the distinction between kinematic and dynamical field ranges. While the SDC is a kinematic statement about the moduli space, i.e., it tracks the change in the low-energy EFT from $p_{0}$ to $p$, it makes no reference to the path connecting them being the true trajectory dictated by the underlying dynamics. In applying the swampland conjectures (e.g., to inflation), however, we are interested in the restriction on the dynamical field range traversed by the true trajectory that solves the equations of motion (EOM). As we will see, the dynamical field range and a geodesic distance traversed in field space by the light field only coincide if the light and heavy sectors evolve separately or when the heavy fields are infinitely heavy. The two field distances deviate from each other precisely because of the hierarchy in Eq. (3), which mandates the heavy fields to be not arbitrarily heavier than the light field of interest. The trajectory can be approximated by a geodesic if there is a sufficiently large mass hierarchy. Our findings do not contradict the SDC [4], which is concerned with the asymptotical behavior as $d\left(p_{0}, p\right) \rightarrow \infty$. However, what we found has a bearing on the refined swampland distance conjecture (RSDC) [12], in which it is argued that $\lambda \sim \mathcal{O}(1)$. We will show explicitly that the constraints on field ranges imposed when $\lambda \sim \mathcal{O}(1)$ for massive axions are obtained from unphysical trajectories. This prescription will agree with the dynamical field range in the presence of a sufficiently large mass hierarchy between the light and heavy sectors, but then the critical field range is also extended.

\section{THE DYNAMICAL FIELD RANGE}

\section{A. The refined swampland conjecture for axions}

Before we present a more critical assessment of the dynamical field range, let us revisit the RSDC constraints for axions [13], in which it is claimed that strong backreaction of the closed-string sector on the kinetic term of an axion constrains its available field range to $\Delta \phi<\mathcal{O}(1) M_{\mathrm{Pl}}$. This claim, if true, excludes the possibility of large-field inflation based on axions, including axion monodromy models $[14,15]$ (and its realizations via the F-term potential in supergravity [16-18]).

The approach used in [13] to impose constraints on the available field range consists of analyzing the trajectory traced by the minima of the heavy fields as we vary an axion, $\varphi$. Then, one should keep all the fields in the action and adjust their values appropriately. This approach is claimed to be a good approximation irrespective of the existence of a mass hierarchy between the sectors. More concretely, for a massive axionic direction defined as a linear combination of certain fields, $\varphi=h^{i} \nu^{i}$, where $h^{i}$ are constants, it is argued that the field range of interest is

$$
\Delta \phi=\int_{\gamma}\left(h_{i} G^{i j}(\varphi) h_{j}\right)^{-1 / 2} d \varphi:=\int_{\gamma} \sqrt{G_{\varphi \varphi}(\varphi)} d \varphi,
$$

where $G^{i j}(\varphi)$ is the inverse of the field space metric and $G_{\varphi \varphi}(\varphi)$ is defined as the kinetic term of the axionic field evaluated along the aforementioned trajectory. One immediately observes that this definition, implicitly, neglects the displacements of the fields not included in the definition of $\varphi$ and then, by default, the trajectory considered is a geodesic in field space. Then, strong backreaction of the closed-string sector on the kinetic term of $\varphi$ implies that the field range (4) grows at best logarithmically, i.e., $\Delta \phi \approx \alpha^{-1} \log (\varphi)$. This is, precisely, the scaling suggested by the SDC. The critical value for the canonically normalized field before the onset of the logarithmic behavior is $\hat{\varphi}_{\text {back }} \sim \alpha^{-1}$. In the text, hatted variables denote canonically normalized fields. Then, one observes that a tower of states becomes exponentially light as $\varphi$ is displaced, in agreement with the SDC (1), if we identify $\alpha \approx \lambda$. It was argued in [13] that $\lambda \sim \mathcal{O}(1)$. This prescription was generalized to include the open-string sector in $[19,20]$, where it was argued that $\lambda$ is proportional to the mass hierarchy between the axion, $\varphi$, and the closedstring sector.

\section{B. Proper field range and validity of the approximation}

We can try to improve the naive estimate of the allowed field range in Eq. (4) by including the kinetic terms of the heavy fields that were previously neglected. We will see under what conditions the naive estimate can be trusted.

The dynamical field range is the one measured along the one-dimensional trajectory defined by the solution of the EOMs. In a multifield scenario, the homogeneous background fields, $\phi_{0}^{a}=\phi_{0}^{a}(t)$, in a spatially flat FLRW spacetime, satisfy

$$
D_{t} \dot{\phi}_{0}^{a}+3 H \dot{\phi}_{0}^{a}+V^{a}=0
$$

where the covariant derivative in field space is defined by $D_{t} X^{a}=\partial_{t} X^{a}+\Gamma_{b c}^{a} \dot{\phi}_{0}^{b} X^{c}$, and $\Gamma_{b c}^{a}$ are the Christoffel symbols derived from the field space metric $G_{a b}$. Here, latin indices $a, b$ denote real coordinates in field space. It is well known that the solutions to the EOMs are tied to two fundamental properties of the system: (1) the existence of mass hierarchies between different sectors and (2) the geometry of the field space metric (we refer the reader to [21-26] for more details). The variation of the scalar fields along the trajectory is defined as $\dot{\phi}_{0}^{2}=G_{a b} \dot{\phi}_{0}^{a} \dot{\phi}_{0}^{b}$. Then, the proper distance traversed along the path parametrized by $t$ is given by 


$$
\Delta \phi=\int \dot{\phi}_{0} d t=\sqrt{G_{a b} \dot{\phi}_{0}^{a} \dot{\phi}_{0}^{b}} d t
$$

To quantify the error of the naive estimate in Eq. (4), we substitute the solutions which minimize the scalar potential in terms of the displaced field, $\bar{\phi}^{\alpha}(\varphi(t))$, into the action to obtain the proper field range (6). Note that greek indices run over all the fields in the theory except $\varphi$. We emphasize here that there is no guarantee that the trajectory given by this procedure is the one that minimizes the action since it is not granted that the EOMs are solved. Nonetheless, this analysis serves as a self-consistent check of whether the kinetic terms for $\bar{\phi}^{\alpha}$ can be neglected. We have also checked numerically that evaluating Eq. (6) with the actual solution to the equations of motion does not change qualitatively our conclusions.

In the presence of strong backreaction, the kinetic terms of the stabilized fields will not be negligible in general. Indeed, we observe that $\dot{\bar{\phi}}^{\alpha}(\varphi(t)) \approx \frac{\partial \bar{\phi}^{\alpha}(\varphi)}{\partial \varphi} \dot{\varphi}$, which implies a proper field range (6), assuming no kinetic mixing:

$$
\Delta \phi=\int \sqrt{G_{\varphi \varphi}(\varphi)+G_{\alpha \beta}(\varphi) \frac{\partial \bar{\phi}^{\alpha}}{\partial \varphi} \frac{\partial \bar{\phi}^{\beta}}{\partial \varphi}} \dot{\varphi} d t .
$$

Note that the term $G_{\alpha \beta}(\varphi) \frac{\partial \bar{\phi}^{\alpha}}{\partial \varphi} \frac{\partial \bar{\phi}^{\beta}}{\partial \varphi}$ is absent in the expression (4) used in $[13,19,20]$. For Eq. (4) to be a good approximation to the allowed field range, we need to ensure

$$
G_{\alpha \beta}(\varphi) \frac{\partial \bar{\phi}^{\alpha}}{\partial \varphi} \frac{\partial \bar{\phi}^{\beta}}{\partial \varphi} \leq \varepsilon G_{\varphi \varphi}(\varphi)
$$

where we can think of $\varepsilon$ as a measure of the accuracy of the approximation or, in other words, a measure of how well the fields that minimize the potential solve the EOMs [27]. If this condition cannot be satisfied along the whole trajectory, there must exist a critical value, $\varphi_{\text {app }}$, for the displaced field beyond which the approximation fails. We define $\varphi_{\text {app }}$ as the solution to the constraint (8) and, as we will illustrate, it will depend on the relative mass hierarchy. For $\hat{\varphi}>\hat{\varphi}_{\text {app }}$ significant corrections to the field range are expected and a multifield analysis is necessary to capture the dynamics. For $\hat{\varphi}<\hat{\varphi}_{\text {app }}$, the single-field approximation is valid. The approximation for the field range (4) is, then, valid within a ball in moduli space centered at the minimum of the potential with radius $\hat{\varphi}_{\text {app }}$. The dynamical field range is thus valid within this approximation up to $\hat{\varphi}_{c}=\min \left\{\hat{\varphi}_{\text {app }}, \hat{\varphi}_{\text {back }}\right\}$. We will check that in the absence of mass hierarchies, $\hat{\varphi}_{\text {app }}<\hat{\varphi}_{\text {back }}$. The real cutoff of the effective theory should then be obtained by solving the full multifield dynamics and, thus, the field range obtained in $[13,19,20]$ will differ from the dynamical field range. On the contrary, the allowed field range is given by $\hat{\varphi}_{\text {back }}$ only if a sufficiently large mass hierarchy is assured. In this regime, corrections to the action generically flatten the potential [10]. Then, one may quantify the backreaction by perturbing the heavy fields around the minimum of the potential. This procedure has been systematically analyzed for $\mathcal{N}=1$ supergravity [11] (see [28] for a shortcut).

\section{Is the trajectory a geodesic?}

We are now in a position to address whether the trajectory which defines the dynamical field range is a geodesic. Recent studies of swampland distances, see, e.g., $[5,29]$, relied on geodesic trajectories in field space for massless axions. We will critically assess under what conditions this assumption holds for massive axions. In multifield setups, it is convenient to define a unit vector tangent to the trajectory which for the present case is

$$
T^{a}:=\frac{\dot{\phi}_{0}^{a}}{\dot{\phi}_{0}} \rightarrow \dot{\phi}_{0} T^{\varphi}=\dot{\varphi}, \quad \dot{\phi}_{0} T^{\alpha}=\frac{\partial \bar{\phi}^{\alpha}}{\partial \varphi} \dot{\varphi} .
$$

As in [30], one may define an orthonormal basis in field space by taking covariant derivatives of the tangent vector, $D_{t} T$. For $\dot{\phi}_{0} \neq 0$, the trajectory obtained by solving the EOMs (5) will be a geodesic in field space if $D_{t} T^{a}=0$. Then, a sufficient condition for the trajectory considered to be a geodesic is

$$
\left(\frac{\partial \bar{\phi}^{\alpha}}{\partial \varphi}\right) \approx 0 \rightarrow\left|D_{t} T\right| \approx 0
$$

Indeed, one may approximate the trajectory by a geodesic when Eq. (8) is satisfied. This is a reasonable approximation if there are large mass hierarchies, and in the absence of nontrivialities coming from the geometry of the field space (see, e.g., [23] for the latter point). Note that the trajectories studied in $[13,19,20]$ are indeed geodesics since (10) is assumed.

\section{AN ILLUSTRATIVE EXAMPLE}

We now try to quantify the statements made in the previous section by analyzing a single-field inflationary model based on D7-branes in the type IIB flux compactification of a toroidal orientifold. We refer the reader to the Supplemental Material [31] for a similar discussion for closed-string models. For ease of comparison, we will focus on an example used in [20] which involves nongeometric fluxes; we refer the reader to that reference. The inflaton candidate, $\varphi$, is the axionic component of the position modulus of a D7-brane. The $\mathcal{N}=1$ supergravity Lagrangian describing this model is given by

$$
\begin{aligned}
K= & -\log \left[(S+\bar{S})(U+\bar{U})-\frac{1}{2}(\Phi+\bar{\Phi})^{2}\right] \\
& -2 \log (U+\bar{U})-3 \log (T+\bar{T}),
\end{aligned}
$$




$$
W=\mathfrak{f}_{0}+3 \mathfrak{f}_{2} U^{2}-h S U-q T U-\mu \Phi^{2}
$$

We note that the mass hierarchy between the axionic field, its saxionic partner $\chi$, and the closed-string sector in the tachyon-free AdS vacuum [32] is given by

$$
\frac{M_{\varphi}}{M_{\text {closed } \chi}} \sim \sqrt{\frac{\mu}{h}} .
$$

Thus, in this example, the only way to generate a hierarchy is to tune the ratio (13). It has been shown in $[19,34,35]$ that tuning $\mu \ll 1$ can be done while satisfying the experimental constraints on the inflationary parameters given by the recent PLANCK data [8]. One should, however, be cautious about this tuning (see the Supplemental Material [31] for more details). As our discussions below depend only on the existence of a mass hierarchy and not on the details of how it is achieved, we will sidestep these challenges and focus on exploring the consequences.

\section{A. The RSDC in different regimes}

To consistently ignore the kinetic terms of the heavy moduli, we should check if Eq. (8) is satisfied. Then, substituting the minima traced by the heavy fields into (8), one arrives at

$$
7 \frac{\hat{\varphi}^{2}}{\hat{\varphi}^{2}+\left(\frac{M_{\text {closed }}}{M_{\varphi}}\right)^{2}} \lesssim \varepsilon\left(\frac{M_{\text {closed }}}{M_{\varphi}}\right)^{2} .
$$

The critical value where the approximation breaks down, $\hat{\varphi}_{\text {app }}$, may be obtained by solving the above expression. Here, we point out that in (8) the backreaction effect of each modulus is weighted by its mass compared to that of the axionic field. In this example, all the stabilized moduli have roughly the same mass and, thus, their effects contribute on equal footing. In a compactification following the Kachru, Kallosh, Linde, and Trivedi (KKLT) [36] or the large volume scenario (LVS) [37] prescriptions, however, there is a hierarchy of scales among the closed-string sectors (see $[18,38]$ for examples). In this case, the RSDC constraint (4) is weaker compared to the constraint imposed by destabilization of the Kähler modulus when a large mass hierarchy between the axion and the complex structure sector is assured (for a geometrical upper bound for the axion range in LVS compactifications, see [39]). Nonetheless, one should use (8) in order to trust the singlefield approximation.

From (14) we see explicitly, as we previously anticipated, that there are two limiting regimes:

Large mass hierarchy. We observe that for $\left(\frac{M_{\text {closed }}}{M_{\varphi}}\right)^{2}>\frac{7}{\varepsilon}$, Eq. (8) is satisfied for all $\hat{\varphi}$ and thus the approach used in the RSDC is valid. The dynamical field range is approximately measured along a geodesic trajectory and thus

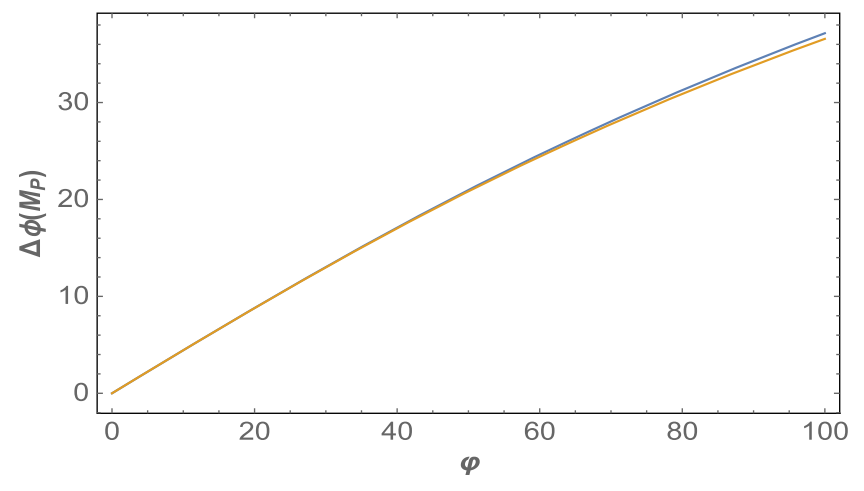

FIG. 1. Field ranges obtained by (7) (blue) and (4) (orange) for $\frac{\mathfrak{f}_{0}}{\mu} \sim 10^{4}$ and $\left.G_{\varphi \bar{\varphi}}\right|_{0} \sim 2 \times 10^{-1}$.

$\Delta \phi=\int \sqrt{G_{a b} \dot{\phi}^{a} \dot{\phi}^{b}} d t \approx \int \sqrt{G_{\varphi \varphi}(\varphi)} d \varphi \sim \frac{M_{\text {closed }}}{M_{\varphi}} \log (\varphi)$.

Then, the critical field range is super-Planckian since $\hat{\varphi}_{c}=\hat{\varphi}_{\text {back }} \sim \frac{M_{\text {closed }}}{M_{\varphi}} M_{\mathrm{Pl}}>1 M_{\mathrm{Pl}}$, in agreement with [20]. We have depicted in Fig. 1 the comparison between the dynamical field range and the geodesic distance.

No mass hierarchy. We observe that for $\frac{M_{\text {closed }}}{M_{\varphi}} \sim 1$, the critical field $\hat{\varphi}_{c}=\hat{\varphi}_{\text {app }} \sim \sqrt{\frac{\varepsilon}{7}} M_{\mathrm{Pl}}<1 M_{\mathrm{Pl}}$, which signals the breakdown of the approximation in Eq. (8) rather than the breakdown of the EFT.

We now compare the RSDC estimate of the field range using Eq. (4) with the measure Eq. (7) in cases where there are no mass hierarchies outside the validity regime we have established in (8). We show in Fig. 2 how the two field ranges deviate from each other, precisely, beyond $\varphi_{\text {app }}$. Then, in this regime one should solve numerically the full multifield dynamics. We have also computed numerically the field range with the actual solution to the EOMs and found that the behavior depicted in Fig. 2 does not change qualitatively. We observe that, asymptotically, for large $\varphi$

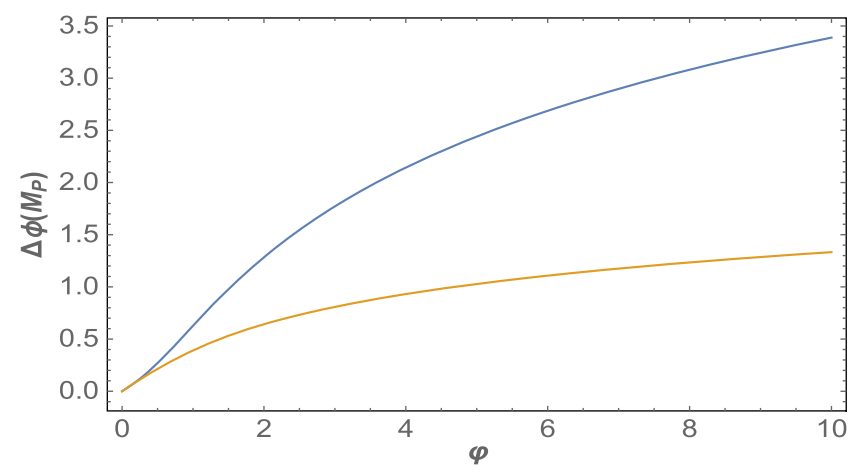

FIG. 2. Field range obtained by (7) (blue) and (4) (orange) for $\frac{\mathfrak{f}_{0}}{\mu} \sim 1$ and $\left.G_{\varphi \bar{\varphi}}\right|_{0} \sim 2 \times 10^{-1}$. In this case $\hat{\varphi}_{\text {app }} \sim 0.1 M_{\mathrm{Pl}}$ for $\varepsilon=0.1$. 
displacements, the dynamical field range grows at best logarithmically, in agreement with the SDC. Finally, our computation shows that the larger the backreaction effects the larger the deviation between the naive approximate (15) and the dynamical field range.

\section{DISCUSSION}

In this paper we have analyzed carefully the viability of the constraints imposed by the RSDC on trans-Planckian field displacements of massive axions. We illustrated our general results with axions in type II compactifications.

One of our findings is that minimizing the scalar potential without taking into account peculiarities of the field space metric and the mass hierarchies involved may lead to inconsistent results. In the examples analyzed in the RSDC literature, the available field range is measured along a geodesic trajectory while neglecting the kinetic terms of the other fields. This is, indeed, a reasonable approximation where the dynamical field range is well approximated by the geodesic distance, if there is a large mass hierarchy and when the backreaction effects are mild. Precisely, in this approximation, the critical field range is extended to a super-Planckian value. In the absence of a mass hierarchy, the approximation breaks down before the onset of the logarithmic behavior that defines the RSDC field range and, then, the sub-Planckian field restriction imposed by the RSDC loses its physical validity. This fact points to one of our premises, which is the importance of analyzing the dynamical field range. In order to constrain field displacements by means of the SDC, it should be applied to physical trajectories. Our findings support this conjecture since the logarithmic behavior of the field range holds asymptotically in all the cases studied, but the concrete point where the EFT suffers a breakdown can only be obtained explicitly by solving the EOMs. It is well known in the context of multifield dynamics that the kinetic terms of the heavy fields can lead to turns in field space. The deviation of the true trajectory from a geodesic, in the context of inflation, can be parametrized by $\eta_{\perp}$ $[21,22,24,25]$.

Given the role of mass hierarchies in defining the dynamical field range, it is important to explore new ways of generating such hierarchies in string compactifications. While ideas such as employing large ratios of flux quanta have been put forth, they seem to be challenging to implement in practice, as can be seen in type IIB settings with nongeometric fluxes [9]. It would be interesting to explore other proposals such as the use of warping for the open-string sector [40] and the use of $g_{s}$ loops for Kähler moduli axions [41]. Finding new ways to generate mass hierarchies in string constructions may hold the key to extending the dynamical field range in quantum gravity. We leave for future work the extension of these findings to generic Calabi-Yau compactifications.

\section{ACKNOWLEDGMENTS}

We thank Fernando Marchesano and Wieland Staessens for discussions. This work is supported in part by the DOE Grant No. DE-SC0017647 and the Kellett Award of the University of Wisconsin.
[1] N. Arkani-Hamed, L. Motl, A. Nicolis, and C. Vafa, The string landscape, black holes and gravity as the weakest force, J. High Energy Phys. 06 (2007) 060.

[2] J. Brown, W. Cottrell, G. Shiu, and P. Soler, Fencing in the swampland: Quantum gravity constraints on large field inflation, J. High Energy Phys. 10 (2015) 023.

[3] J. Brown, W. Cottrell, G. Shiu, and P. Soler, On axionic field ranges, loopholes and the weak gravity conjecture, J. High Energy Phys. 04 (2016) 017.

[4] H. Ooguri and C. Vafa, On the geometry of the string landscape and the swampland, Nucl. Phys. B766, 21 (2007).

[5] T. W. Grimm, E. Palti, and I. Valenzuela, Infinite distances in field space and massless towers of states, J. High Energy Phys. 08 (2018) 143.

[6] B. Heidenreich, M. Reece, and T. Rudelius, Emergence and the Swampland Conjectures, Phys. Rev. Lett. 121, 051601 (2018).

[7] D. H. Lyth, What Would We Learn by Detecting a Gravitational Wave Signal in the Cosmic Microwave Background Anisotropy?, Phys. Rev. Lett. 78, 1861 (1997).
[8] P. A. R. Ade et al. (Planck Collaboration), Planck 2015 results. XX. Constraints on inflation, Astron. Astrophys. 594, A20 (2016).

[9] R. Blumenhagen, D. Herschmann, and E. Plauschinn, The challenge of realizing F-term axion monodromy inflation in string theory, J. High Energy Phys. 01 (2015) 007.

[10] X. Dong, B. Horn, E. Silverstein, and A. Westphal, Simple exercises to flatten your potential, Phys. Rev. D 84, 026011 (2011).

[11] W. Buchmuller, E. Dudas, L. Heurtier, A. Westphal, C. Wieck, and M. W. Winkler, Challenges for large-field inflation and moduli stabilization, J. High Energy Phys. 04 (2015) 058.

[12] D. Klaewer and E. Palti, Super-Planckian spatial field variations and quantum gravity, J. High Energy Phys. 01 (2017) 088.

[13] F. Baume and E. Palti, Backreacted axion field ranges in string theory, J. High Energy Phys. 08 (2016) 043.

[14] E. Silverstein and A. Westphal, Monodromy in the CMB: Gravity waves and string inflation, Phys. Rev. D 78, 106003 (2008). 
[15] L. McAllister, E. Silverstein, and A. Westphal, Gravity waves and linear inflation from axion monodromy, Phys. Rev. D 82, 046003 (2010).

[16] F. Marchesano, G. Shiu, and A. M. Uranga, F-term axion monodromy inflation, J. High Energy Phys. 09 (2014) 184.

[17] R. Blumenhagen and E. Plauschinn, Towards universal axion inflation and reheating in string theory, Phys. Lett. B 736, 482 (2014).

[18] A. Hebecker, S. C. Kraus, and L. T. Witkowski, D7-brane chaotic inflation, Phys. Lett. B 737, 16 (2014).

[19] I. Valenzuela, Backreaction issues in axion monodromy and Minkowski 4-forms, J. High Energy Phys. 06 (2017) 098.

[20] R. Blumenhagen, I. Valenzuela, and F. Wolf, The swampland conjecture and F-term axion monodromy inflation, J. High Energy Phys. 07 (2017) 145.

[21] S. G. Rubin, Effect of massive fields on inflation, Pis'ma Zh. Eksp. Teor. Fiz. 74, 275 (2001) [JETP Lett. 74, 247 (2001)].

[22] A. J. Tolley and M. Wyman, The gelaton scenario: Equilateral non-Gaussianity from multi-field dynamics, Phys. Rev. D 81, 043502 (2010).

[23] A. Achucarro, J.-O. Gong, S. Hardeman, G. A. Palma, and S. P. Patil, Mass hierarchies and non-decoupling in multiscalar field dynamics, Phys. Rev. D 84, 043502 (2011).

[24] G. Shiu and J. Xu, Effective field theory and decoupling in multi-field inflation: An illustrative case study, Phys. Rev. D 84, 103509 (2011).

[25] A. Achucarro, V. Atal, S. Cespedes, J.-O. Gong, G. A. Palma, and S. P. Patil, Heavy fields, reduced speeds of sound and decoupling during inflation, Phys. Rev. D 86, 121301 (2012).

[26] X. Gao, D. Langlois, and S. Mizuno, Influence of heavy modes on perturbations in multiple field inflation, J. Cosmol. Astropart. Phys. 10 (2012) 040.

[27] For consistent decoupling between sectors, $\varepsilon=0$.

[28] A. Landete, F. Marchesano, and C. Wieck, Challenges for D-brane large-field inflation with stabilizer fields, J. High Energy Phys. 09 (2016) 119.
[29] R. Blumenhagen, D. Klwer, L. Schlechter, and F. Wolf, The refined swampland distance conjecture in Calabi-Yau moduli spaces, J. High Energy Phys. 06 (2018) 052.

[30] S. G. Nibbelink and B. J. W. van Tent, Scalar perturbations during multiple field slow-roll inflation, Classical Quantum Gravity 19, 613 (2002).

[31] See Supplemental Material at http://link.aps.org/ supplemental/10.1103/PhysRevD.98.066012 for a discussion of the refined swampland distance conjecture for closed-string moduli axions and for more details on the mass hierarchies in presence of the open-string sector.

[32] For the sake of simplicity, we consider a constant uplift term. We have checked that the final results do not differ quantitatively if one considers an F-term uplift coming from a nilpotent Goldstino [33].

[33] S. Ferrara, R. Kallosh, and A. Linde, Cosmology with nilpotent superfields, J. High Energy Phys. 10 (2014) 143.

[34] S. Bielleman, L. E. Ibanez, F. G. Pedro, I. Valenzuela, and C. Wieck, Higgs-otic inflation and moduli stabilization, J. High Energy Phys. 02 (2017) 073.

[35] A. Landete, F. Marchesano, G. Shiu, and G. Zoccarato, Flux flattening in axion monodromy inflation, J. High Energy Phys. 06 (2017) 071.

[36] S. Kachru, R. Kallosh, A. D. Linde, and S. P. Trivedi, De Sitter vacua in string theory, Phys. Rev. D 68, 046005 (2003).

[37] V. Balasubramanian, P. Berglund, J. P. Conlon, and F. Quevedo, Systematics of moduli stabilisation in Calabi-Yau flux compactifications, J. High Energy Phys. 03 (2005) 007.

[38] L. E. Ibanez, F. Marchesano, and I. Valenzuela, Higgs-otic inflation and string theory, J. High Energy Phys. 01 (2015) 128.

[39] M. Cicoli, D. Ciupke, C. Mayrhofer, and P. Shukla, A geometrical upper bound on the inflaton range, J. High Energy Phys. 05 (2018) 001.

[40] D. Escobar, A. Landete, F. Marchesano, and D. Regalado, Large field inflation from D-branes, Phys. Rev. D 93, 081301 (2016).

[41] M. Cicoli, K. Dutta, and A. Maharana, N-flation with hierarchically light axions in string compactifications, J. Cosmol. Astropart. Phys. 08 (2014) 012. 